Original

\title{
Association between headache and temporomandibular joint disorders in children and adolescents
}

\author{
Luciana P. Branco1), Tatiana O. Santis2), Thays A. Alfaya ${ }^{3)}$, Camila H. L. Godoy2), \\ Yara D. Fragoso4), and Sandra K. Bussadori ${ }^{5)}$ \\ 1)Undergraduate Program in Medical School, University of Metropolitana de Santos (UNIMES), \\ Santos, SP, Brazil \\ 2)Postgraduate Program in Rehabilitation Sciences, University of Nove de Julho (UNINOVE), \\ São Paulo, SP, Brazil \\ 3)Postgraduate Program in General Dentistry, University of Federal Fluminense (UFF), Niterói, RJ, Brazil \\ 4)Department of Neurology, Medical School, University of Metropolitana de Santos (UNIMES), \\ Santos, SP, Brazil \\ 5)Rehabilitation Sciences Postgraduate Program, University of Nove de Julho (UNINOVE), \\ São Paulo, SP, Brazil
}

(Received August 6, 2012; Accepted December 25, 2012)

\begin{abstract}
The aim of the present study was to assess the relationship between temporomandibular joint disorder (TMJD) and headache in children and adolescents. A prospective cross-sectional cohort study was carried out involving 93 children and adolescents $(6$ to 14 years of age) at the outpatient service of a dental school. All participants underwent a clinical examination involving Axis 1 of the Research Diagnostic Criteria for Temporomandibular Disorders, along with a characterization of headache and an anthropometric evaluation. Statistical analysis involved the chi-squared test for quantitative variables and the Student's t-test, ANOVA and Tukey's test for quantitative data. An adjusted logistic regression model was used to determine significant associations among gender, age, TMJD and headache. Mild TMJD was identified in $35.8 \%$ of the sample and was not associated the presence of headache. Moderate TMJD was found in $\mathbf{2 5 . 8 \%}$ of patients and severe TMJD was found in $11.8 \%$; both forms of TMJD were associated with headache. A significant correlation was found between the intensity of TMJD and the risk of headache. The present findings demonstrate a positive
\end{abstract}

Correspondence to Dr. Thays Almeida Alfaya, R. Doutor Calandrine 235 A, São Gonçalo, 24755.160 Rio de Janeiro, Brazil E-mail: thalfaya@gmail.com correlation between TMJD and headache in children and adolescents, independently of gender and age. (J Oral Sci 55, 39-43, 2013)

Keywords: headache; temporomandibular joint disorder; child, adolescent.

\section{Introduction}

Headache is a highly prevalent heterogeneous disorder classified as either primary (when no other condition justifies the symptom) or secondary (when a symptom of another health condition) (1). Primary headache, such as migraine and tension-type headache, affects Brazilian children with a prevalence rate of $12.3 \%$ and $4.2 \%$, respectively (2). Although the origin or worsening of headache in children and adolescents may also be related to temporomandibular joint disorder (TMJD), few studies have addressed this association.

TMJD is a collective term that embraces a number of clinical problems that involve the temporomandibular joint and related structures. Headache is one of the major symptoms reported by individuals with TMJD (3). A recent Finnish study found that TMJD affected one third of a large population of children, often associated with pain in other parts of the body (3). A Brazilian study validating an oral health-related quality of life questionnaire 
Table 1 Characteristics of the sample $(n=93)$

\begin{tabular}{lc}
\hline & $n(\%)$ \\
\cline { 2 - 2 } Gender & $51(54.8)$ \\
Female & $42(45.2)$ \\
Male & \\
Type of bite & $12(12.9)$ \\
Anterior open bite & $1(1.1)$ \\
Anterior open bite + Right unilateral posterior crossbite & $6(6.5)$ \\
Anterior open bite + Left unilateral posterior crossbite & $1(1.1)$ \\
Anterior crossbite & $1(1.1)$ \\
Anterior crossbite + Left unilateral posterior crossbite & $7(7.5)$ \\
Right unilateral posterior crossbite & $3(3.2)$ \\
Bilateral posterior crossbite & $3(3.2)$ \\
Left unilateral posterior crossbite & $59(63.4)$ \\
Without malocclusion & \\
TMJDs & $33(35.5)$ \\
Mild & $24(25.8)$ \\
Moderate & $11(11.8)$ \\
Severe & $25(26.9)$ \\
TMD absent & \\
Headache & $42(45.2)$ \\
No & $51(54.8)$ \\
Yes & \\
Occlusal wear & $84(90.3)$ \\
No & $9(9.7)$ \\
Yes &
\end{tabular}

for children also reported the disrupting effect of TMJD on children and pre-adolescents (4). When subgroups are assessed, girls and children/adolescents with posterior unilateral crossbite are at greater risk of developing craniofacial pain (5).

The study of headache in children and adolescents with and without TMJD poses a challenge to multidisciplinary teams caring for this population. The aim of the present study was to assess the prevalence and type of TMJD in children and adolescents referred to the dentist due to headache.

\section{Methods}

A prospective cross-sectional cohort study was carried out involving 93 children and adolescents (six to 14 years of age) at the dental clinic of the Universidade Metropolitana de Santos (Brazil). This study received approval from the Human Research Ethics Committee of the university (process number 056/2011). Adults responsible for the underage patients signed a statement of informed consent allowing the evaluations.

The inclusion criteria were the presence of second deciduous molars in children aged six to ten years and the first permanent molar in those aged 11 to 14 years. Children and adolescents undergoing chronic medical, orthodontic or psychological treatment and those with craniofacial deformities were excluded.

The Helkimo Index was administered to all partici- pants who met the eligibility criteria for the evaluation of TMJDs. This index is more easily understood by individuals in the age group studied and makes it possible to classify individuals into severity categories based on the clinical signs of TMJD. The index is subdivided into five items, with three possible scores for each item $(0,1$, and 5 ), depending on the clinical evaluation. The total allows classification into categories: absence of signs of TMJD (0 points), mild TMJD (1 to 4 points), moderate TMJD (5 to 9 points) and severe TMJD (10 to 25 points) (6-10).

Clinical examinations were performed using Axis 1 of the Research Diagnostic Criteria for Temporomandibular Disorders (11). The evaluation was performed by the same dentist, familiar with this assessment tool. The clinical examination included a comprehensive assessment of teeth and dental occlusion, palpation of the temporomandibular joint and the trapezius, sternocleidomastoid, temporal, masseter, digastric and medial pterygoid muscles. Analysis of mandibular movements was carried out with the aid of a digital caliper (Mitutoyo, Kawasaki, Japan) for the assessment of maximum oral opening and lateral excursion. Joint noises, anthropometric evaluation and the presence of headache were investigated individually.

Statistical analysis was performed using the SAS program for Windows (v. 9.2) and involved the chisquared test for quantitative variables and the Student's $t$-test, ANOVA and Tukey's test for quantitative data. An 
Table 2 Association between headache and other variables $(n=93)$

\begin{tabular}{lrrr}
\hline & \multicolumn{2}{c}{ Headache } & \multirow{2}{*}{$P$ value } \\
\cline { 2 - 3 } & Yes & No & \\
\hline Gender & & & 0.6853 \\
Female & 24 & 27 & \\
Male & 18 & 24 & \multirow{2}{*}{0.7170} \\
Type of bite & & & \\
Anterior open bite & 7 & 5 & \\
Anterior open bite + Right unilateral posterior crossbite & 0 & 1 & \\
Anterior open bite + Left unilateral posterior crossbite & 3 & 3 & \\
Anterior crossbite & 0 & 1 & \\
Anterior crossbite + Left unilateral posterior crossbite & 1 & 0 & \\
Right unilateral posterior crossbite & 4 & 3 & \\
Bilateral posterior crossbite & 1 & 2 & \\
Left unilateral posterior crossbite & 1 & 2 & \\
Without malocclusion & 25 & 34 & \multirow{2}{*}{0.0146} \\
TMJDs & & & \\
Absent & 15 & 10 & \\
Mild & 18 & 15 & \\
Moderate & 8 & 16 & \\
Severe & 1 & 10 & \\
Occlusal wear & & & 0.9317 \\
No & 38 & 46 & \\
Yes & 4 & 5 & \\
\hline
\end{tabular}

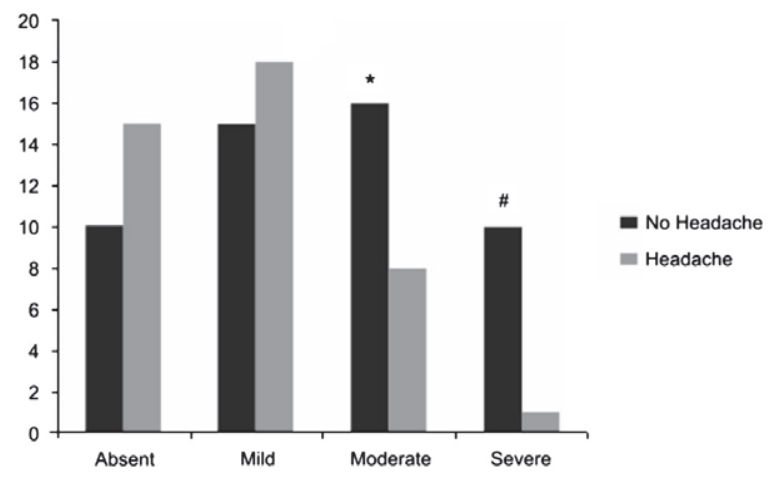

Fig. 1 Association between headache and TMJD $(* P=0.04 ; \# P=0.001)$.

adjusted logistic regression model was used to determine significant associations between headache (dependent variable) and gender, age and TMJD (explanatory variables). For all tests, the significance level was set to $5 \%$ $(P<0.05)$.

\section{Results}

Ninety-three individuals aged 6 to 14 years were evaluated. Females accounted for $54.8 \%$ of the total sample. Table 1 displays the general demographic data.

The clinical examination revealed an absence of malocclusion in $63.4 \%$ of the participants $(n=59)$. Cases of malocclusion involved anterior open bite $(n=12)$ and anterior open bite associated with left $(n=6)$ and right $(n=6)$ posterior crossbite. Occlusal wear was found in nine patients.
When the group of patients was divided into younger (6 to 10 years) and older (11 to 16 years) subgroups, no significant difference was found regarding the presence of TMD and/or headache. Likewise, no statistically significant gender differences were found regarding the presence of TMD and/or headache (Table 2).

Mild TMJD was found in $35.5 \%$ of the patients $(n=$ $33)$, moderate TMJD in $25.8 \%(n=24)$ and severe TMJD in $11.9 \%(n=11)$. Headache was a major complaint in $54.9 \%(n=51)$ of the children and adolescents and was not associated with age, gender, type of bite or occlusal wear. Table 2 summarizes the significant associations between TMJD and headache. Absence of TMJD was not associated with headache $(P=0.26)$. The same was true for mild TMD $(P=0.622)$. However, headache was significantly associated with moderate $(P=0.04)$ and severe TMJD $(P=0.001)$ (Fig. 1$)$. Logistic regression analysis revealed that children or adolescents with moderate TMJD had a threefold greater chance of having headache and that those with severe TMJD had a 16-fold greater chance of having headache in comparison to those without TMJD (Table 3).

\section{Discussion}

The high prevalence of headache and TMJD observed in the children and adolescents assessed in the present study is likely the result of selection bias, as the patients actively sought dental consultations and were not selected from the general or school population. Therefore, no conclusions can be drawn regarding the prevalence of signs and 
Table 3 Adjustment of the logistic model for presence of headache with TMJD and sex

\begin{tabular}{lcccc}
\hline Variable & Estimate & Standard error & $P$ value & OR (95\% CI) \\
\hline Constant & 0.6332 & 0.3174 & 0.0459 & - \\
TMJDs Mild & -0.8226 & 0.4041 & 0.0418 & $0.073(0.008-0.671)$ \\
TMJDs Moderate & 0.0806 & 0.4398 & 0.8547 & $0.200(0.020-1.739)$ \\
TMJDs Absent & -1.0021 & 0.4282 & 0.0172 & $0.062(0.007-0.569)$ \\
TMJDs Severe & - & - & - & 1.00 \\
Female & -0.1813 & 0.2247 & 0.4197 & $0.696(0.288-0.679)$ \\
Male & - & - & - & 1.00 \\
\hline
\end{tabular}

symptoms in these patients.

The present study revealed a significant association between TMJD and headache in children and adolescents. As pointed out previously by other authors, the dysfunctional stomatognathic system may explain the pain reported by these patients (12-15). Although such findings are not uncommon in adults and have been demonstrated in large population-based studies $(12,15)$, similar studies involving younger individuals have been rare. In the present study, no significant association was found between gender and the presence of TMJD and headache. However, females tend to exhibit a greater frequency of TMJD symptoms than males during adolescence (16), which may be attributed to the greater sensitivity and pain upon palpation of the temporomandibular joint and muscles of the stomatognathic system in females (17).

An interesting aspect of the present study was the lack of a direct association between TMD/headache and age or gender, which is in agreement with findings reported in a large study by Keeling and co-workers (18). However, this relationship has not always been investigated or described (19) and some studies have only cited age as exerting an influence on the association between these clinical conditions $(20,21)$. Nevertheless, a recent large study by Tecco et al. reported a significant association between TMD/headache and patient characteristics, such as female gender, young age, and the presence of posterior unilateral crossbite (22).

Although a number of studies have described a relationship between malocclusion and TMJD/headache, the present investigation found no significant association between these parameters. A recent study by Troeltzsch and co-authors found that only displacements greater than $3 \mathrm{~mm}$ were associated to headache (15). Castelo and co-workers also found that, with the exception of atypical swallowing, no parafunctional or occlusal disturbances were associated with TMJD in children in the primary dentition phase (23). On the other hand, Thillander and collaborators found an association between TMJD/pain and posterior crossbite, anterior open bite, Angle Class
III malocclusion and extreme maxillary overjet (16). Considering the principle that parafunctional habits and stress-related events play a fundamental role in the etiology of both TMJD and headache (24-26), further studies are needed to demonstrate and clarify this association in adolescents.

The results of the present study and findings reported in the literature underscore the need for more detailed studies on the subject. It is not rare to find physicians and dentists who are unaware of the complexity of this issue while treating children and adolescents with headache (14). An analysis of primary headache, such as migraine and tension-type headache (1), in the pediatric population seeking dental care is warranted. Large Finnish studies have found that children with migraine had more signs and symptoms of TMJD than children without migraine or with tension-type headache $(27,28)$. The association between tension-type headache and TMJD is even more complex, since these conditions may overlap (14). Indeed, disorders of the temporomandibular joint and adjacent structures are possible triggering factors for tension-type headache $(14,29)$. The common mechanisms for this association may be the presence of central facilitation of nociceptive inputs (13).

The present study identified a clear association between TMJD severity and headache in children and adolescents, independently of gender and age, as individuals with moderate to severe forms of this disorder respectively had a threefold and 16-fold greater chance of having headache in comparison to those without TMD.

\section{References}

1. Headache Classification Subcommittee (2004) The international classification of headache disorders: 2nd edition. Cephalalgia 24, Suppl 1, 9-160.

2. Arruda MA, Guidetti V, Galli F, Albuquerque RC, Bigal ME (2010) Migraine, tension-type headache, and attention-deficit/ hyperactivity disorder in childhood: a population-based study. Postgrad Med 122, 18-26.

3. Vierola A, Suominen AL, Ikavalko T, Lintu N, Lindi V, Lakka $\mathrm{HM}$ et al. (2012) Clinical signs of temporomandibular disorders and various pain conditions among children 6 to 8 years 
of age: the PANIC study. J Orofac Pain 26, 17-25.

4. Barbosa TS, Leme MS, Castelo PM, Gavião MB (2011) Evaluating oral health-related quality of life measure for children and preadolescents with temporomandibular disorder. Health Qual Life Outcomes 9, 32.

5. Tecco S, Festa F (2010) Prevalence of signs and symptoms of temporomandibular disorders in children and adolescents with and without crossbites. World J Orthod 11, 37-42.

6. Chaves TC, Oliveira AS, Grossi DB (2008) Main instruments for assessing temporomandibular disorders, part I: indices and questionnaires; a contribution to clinicians and researchers. Fisioter Pesqui 15, 92-100.

7. Ardizone I, Celemin A, Aneiros F, del Rio J, Sanchez T, Moreno I (2010) Electromyographic study of activity of the masseter and anterior temporalis muscles in patients with temporomandibular joint (TMJ) dysfunction: comparison with the clinical dysfunction index. Med Oral Patol Oral Cir Bucal 15, e14-19.

8. Bourzgui F, Sebbar M, Nadour A, Hamza M (2010) Prevalence of temporomandibular dysfunction in orthodontic treatment. Int Orthod 8, 386-398.

9. Leuin SC, Frydendall E, Gao D, Chan KH (2011) Temporomandibular joint dysfunction after mandibular fracture in children: a 10-year review. Arch Otolaryngol Head Neck Surg 137, 10-14.

10. Perillo L, Femminella B, Farronato D, Baccetti T, Contardo L, Perinetti G (2011) Do malocclusion and Helkimo Index $\geq$ 5 correlate with body posture? J Oral Rehabil 38, 242-252.

11. Dworkin SF, LeResche L (1992) Research diagnostic criteria for temporomandibular disorders: review, criteria, examinations and specifications, critique. J Craniomandib Disord 6, 301-355.

12. Cooper BC, Kleinberg I (2007) Examination of a large patient population for the presence of symptoms and signs of temporomandibular disorders. Cranio 25, 114-126.

13. Gonçalves DA, Camparis CM, Speciali JG, Franco AL, Castanharo SM, Bigal ME (2011) Temporomandibular disorders are differentially associated with headache diagnoses: a controlled study. Clin J Pain 27, 611-615.

14. Özkan NC, Özkan F (2011) The relationship of temporomandibular disorders with headaches: a retrospective analysis. Agri 23, 13-17.

15. Troeltzsch M, Troeltzsch M, Cronin RJ, Brodine AH, Frankenberger R, Messlinger K (2011) Prevalence and association of headaches, temporomandibular joint disorders, and occlusal interferences. J Prosthet Dent 105, 410-417.

16. Thilander B, Rubio G, Pena L, de Mayorga C (2002) Prevalence of temporomandibular dysfunction and its association with malocclusion in children and adolescents: an epidemiologic study related to specified stages of dental development.
Angle Orthod 72, 146-154.

17. Dao T, LeResche L (2000) Gender difference in pain. J Orofac Pain 14, 169-184.

18. Keeling SD, McGorray S, Wheeler TT, King GJ (1994) Risk factors associated with temporomandibular joint sounds in children 6 to 12 years of age. Am J Orthod Dentofacial Orthop 105, 279-287.

19. Bertoli FM, Antoniuk SA, Bruck I, Xavier GR, Rodrigues DC, Losso EM (2007) Evaluation of the signs and symptoms of temporomandibular disorders in children with headaches. Arq Neuropsiquiatr 65, 251-255.

20. Liljeström MR, Le Bell Y, Laimi K, Anttila P, Aromaa M, Jämsä $T$ et al. (2008) Are signs of temporomandibular disorders stable and predictable in adolescents with headache? Cephalalgia 28, 619-625.

21. Carra MC, Huynh N, Morton P, Rompré PH, Papadakis A, Remise C et al. (2011) Prevalence and risk factors of sleep bruxism and wake-time tooth clenching in a 7- to 17-yr-old population. Eur J Oral Sci 119, 386-394.

22. Tecco S, Crincoli V, Di Bisceglie B, Saccucci M, Macrí M, Polimeni A et al. (2011) Signs and symptoms of temporomandibular joint disorders in Caucasian children and adolescents. Cranio 29, 71-79.

23. Castelo PM, Gavião MB, Pereira LJ, Bonjardim LR (2005) Relationship between oral parafunctional/nutritive sucking habits and temporomandibular joint dysfunction in primary dentition. Int J Paediatr Dent 15, 29-36.

24. Gavish A, Halachmi M, Winocur E, Gazit E (2000) Oral habits and their association with signs and symptoms of temporomandibular disorders in adolescent girls. J Oral Rehabil 27, 22-32.

25. Waldie KE (2001) Childhood headache, stress in adolescence, and primary headache in young adulthood: a longitudinal cohort study. Headache 41, 1-10.

26. Winocur E, Littner D, Adams I, Gavish A (2006) Oral habits and their association with signs and symptoms of temporomandibular disorders in adolescents: a gender comparison. Oral Surg Oral Med Oral Pathol Oral Radiol Endod 102, 482-487.

27. Liljeström MR, Jämsä A, Le Bell Y, Alanen P, Anttila P, Metsähonkala L et al. (2001) Signs and symptoms of temporomandibular disorders in children with different types of headache. Acta Odontol Scand 59, 413-417.

28. Liljeström MR, Le Bell Y, Anttila P, Aromaa M, Jämsä T, Metsähonkala L et al. (2005) Headache children with temporomandibular disorders have several types of pain and other symptoms. Cephalalgia 25, 1054-1060.

29. Rosted P, Jørgensen A, Bundgaard M (2010) Temporomandibular dysfunction can contribute to aggravation of tension-type headache: a case report. Acupunct Med 28, 154-155. 